\section{Clonal diversity in glioblastoma: is it clinically relevant?}

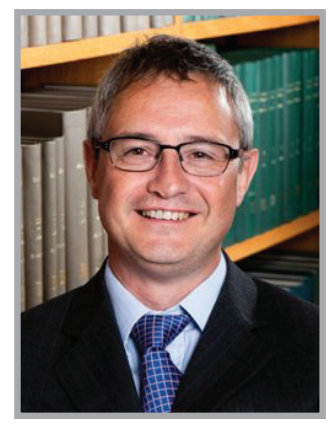

\author{
"Serial tumor sampling, single-cell genomics and \\ quantitative imaging are all available technologies, \\ but their integration into current pathways of care \\ will require a paradigm shift in the clinical \\ management of patients with glioblastoma.”
}

\section{Colin Watts ${ }^{*, 1} \&$ Sara GM Piccirillo 2}

Glioblastoma (GBM), the most common primary brain cancer, is characterized by inter- and intra-patient genomic and histopathological diversity, arising from the complex dynamics that underpin its development. This diversity generates different patterns of clinical behavior whose common feature is the rapid emergence of treatment resistance. All clinicians treating cancer recognize marked variation in tumor behavior and response to therapy between patients with the same tumor type. Multiple factors contribute to this variability, including the tumor environment, pharmacodynamics and heterogeneity within (intratumor) and between (intertumor) individual patients [1-4]. Tumor heterogeneity refers to subpopulations of cells with distinct genotypes and phenotypes that may display divergent biological behaviors within a primary tumor or between tumors of the same histological subtype.

It is argued that tumors could be regarded as complex ecosystems in which even a minor subpopulation may influence the growth of the whole [1]. A clonal evolutionary model of cancer, based on
Darwinian principles of natural selection, postulates that cancer cells accumulate genetic alterations allowing selective pressures to favor the survival of subpopulations with a biological fitness advantage. Clonal evolution as a model for tumor progression has been demonstrated in several cancers, including hematological cancers, pancreatic cancer, renal cell carcinoma and breast cancer [1].

Clonal evolution as a basis for glioma progression was evidenced by early molecular genetic studies of clinical specimens [5,6]. A more detailed analysis has been made possible by the advent of high-throughput next-generation sequencing technologies that provided the opportunity to systematically detect somatic mutations and transcriptional changes on a large scale $[7,8]$. Mapping of genetic alterations onto the major pathways implicated in glioblastoma has identified an interconnected network of aberrations in the p53, $\mathrm{Rb}$ and receptor tyrosine kinase (RTK) signaling pathways. A tendency to mutual exclusivity of genetic alterations within each pathway was observed suggesting that deregulation of one element within

\section{KEYWORDS}

- brain • cancer • cancer stem cells $\bullet$ clinical trials $\bullet$ evolution - genomics • glioblastoma

- sampling bias • therapy

*Department of Clinical Neurosciences, Division of Neurosurgery, University of Cambridge, Box 167, Addenbrooke's Hospital, Hills Road, Cambridge, CB2 0QQ, UK

2John van Geest Centre for Brain Repair, Department of Clinical, Neurosciences, University of Cambridge, Cambridge, CB2 OPY, UK

*Author for correspondence: Tel.: +44 122333 6946; cw209@cam.ac.uk 
“Clonal evolution as a basis for glioma progression was evidenced by early molecular genetic studies of clinical specimens." the pathway relieves the selective pressure for additional mutations. Furthermore $74 \%$ of samples harbored aberrations in all three pathways. These data suggest that deregulation of each of these pathways represents a core selective pressure in the evolution of GBM [9].

A critical drawback of large cohort studies is that they are based on cross-sectional data from single samples and therefore suffer from important sampling biases [10]. The genomic readout from single biopsies may not reflect the variegated genetics of the tumor as a whole and may obscure its underlying evolutionary dynamics. For instance, it has been shown that treatment resistance can be driven by very rare subclones [11], which could not have been detected in single samples at the current level of resolution. In GBM, analysis of archival material using FISH and array comparative genomic hybridization has revealed the simultaneous amplification of up to three different RTKs (EGFR, MET, PDGFRA) [3] in mutually exclusive subpopulations in individual tumors. These mosaic amplifications were observed in $16 / 350(4.5 \%)$ of the GBM tumors. Similarly, analysis of the TCGA dataset identified 34/463 (7.3\%) of GBM patients with focal amplification of two or more RTKs [2]. Dual-color FISH on eight samples with EGFR and PDGFRA amplification identified mutually exclusive subpopulations. Simultaneous inhibition of EGFR and PDGFRA was necessary for abrogation of PI3K activity in a mixed population.

In several cancers, clinically tractable biomarkers occur in the early, preclinical stages of development and are ubiquitous somatic aberrations homogeneously distributed throughout the tumor. For example, somatic mutations in RAS, BRAF and PIK3CA in colorectal cancer, $V H L$ in renal carcinoma and TP53 in triplenegative breast cancer all appear to be early driver events [12]. In glial cancer mutations in the genes encoding isocitrate dehydrogenase 1 and 2 represent early driver events in several subsets of glioma including secondary glioblastoma [13].

Although targeting specific mutations is the goal of personalized medicine, such a strategy in an evolutionary advanced and clonally diverse disease such as glioblastoma will inevitably select for resistant clones. Several studies suggest that treatment resistant cells may already exist in chemo-naive leukemia patients and our group has shown pre-existing resistance to the alkylating agent temozolomide in chemonaive glioblastoma patients [14]. In spatially segregated tumor, initiating cell populations, we observed chemoresistance that could not be abrogated by supramaximal drug concentrations. Furthermore, resistance was present in both MGMT methylated and unmethylated chemo-naive tumors. More sophisticated studies in multiple myeloma and non-small-cell lung cancer have shown that the subclone that may ultimately define therapeutic response and clinical outcome may be present at very low frequency at diagnosis and that dynamic changes in the subclonal architecture of the tumor may change and compete with each other for dominance during the disease course and through different lines of treatment [12].

Intratumor heterogeneity and clonal diversity pose a formidable challenge for personalized management of patients with GBM. Spatial heterogeneity in glioblastoma has been demonstrated in which the RTKs MET, PDGFRA and EGFR are amplified and expressed in a mutually exclusive manner in intermingled cells arising from a common progenitor [3]. Tumor propagating cells derived from the same tumor with heterogeneous EGFR and PDGFRA amplification required inhibition of both oncogenes to optimize growth inhibition through abrogation of PI3K activity [2]. Most patients comprise multiple transcriptional subtypes within the same tumor and contain multiple coexisting cell lineages with variegated evolution of amplification of EGFR and PDGFRA posing further challenges for the development of personalized therapeutics for glioblastoma patients.

The relationship between clonal heterogeneity and outcome and the clinical significance of subclonal driver mutations is only beginning to be explored across cancers. In chronic lymphocytic leukemia, head and neck cancer and nonsmall-cell lung cancer, the presence of subclonal drivers and clonal diversity was associated with a more aggressive clinical phenotype and poor outcomes [12]. In glioblastoma analysis of intratumor heterogeneity at the single-cell level revealed that tumors harboring more diverse transcriptional subtypes were associated with worse survival [15]. Epigenetic factors contribute an additional layer of complexity. For example, in adult glioblastoma, DNA hypermethylation is associated with improved survival within the proneural subgroup and is strongly associated with isocitrate dehydrogenase 1 mutations [9]. 
These data suggest that the inherent instability and plasticity of the cancer genome is permissive for dynamic clonal and subclonal evolution through the gain and loss of distinct genetic aberrations that occur during the course of disease progression. For example, single cell analysis of GBM patients treated with EGFR inhibitors reveals that tumor cells reversibly upregulate and suppress mutant EGFR expression. The result is a dynamic equilibrium established between distinct cellular phenotypes, which optimize tumor growth [16].

The standard of care for the subset of GBM patients suitable for radical treatment remains radiotherapy with concomitant and adjuvant temozolomide [17]. There is increasing recognition that such therapy may itself act as an exogenous source of genome instability. For example, inactivating mutations of the MSHG gene are associated with resistance to alkylating chemotherapy leading to tumor progression on temozolomide in a subset of patients [18]. Temozolomide treatment has also been shown to increase mutations, usually in the form of $\mathrm{C}>\mathrm{T}$ base transitions, leaving a specific mutational signature and more recently comparison of the somatic landscape of paired primary and recurrent GBM found that progression was strongly associated with hypermutation [19]. The hypermutated tumors had been treated with temozolomide and many temozolomideinduced mutations were found in putative driver genes including $R B 1$ and $C D K N 2 A$. Genetic alterations in the p53 pathway appear to be predictive of a high number of subclonal mutations and both linear and divergent patterns of progression have been identified [20]. Thus, treatment also acts as a selection barrier, shaping the evolutionary trajectory of glioblastoma toward a more aggressive phase with seeding of the recurrence from recent ancestors present in the primary disease or cells that diverged early during tumor evolution.

These relationships between clonal diversity and clinical outcome highlight the need to develop sensitive clinical tools to quantify intratumor heterogeneity and detect and monitor the dynamics of subclonal events during treatment. This is likely to prove challenging in glioblastoma because it will require methods capable of integrating clinical and bioinformatics data obtained in ways that control for spatial and temporal evolutionary dynamics $[4,15,20]$. Defining the number of driver events, their subclonal nature and their potential epistatic relationships will likely require spatial and longitudinal sequencing (and hence biosampling) of individual patients. Serial tumor sampling, single-cell genomics and quantitative imaging are all available technologies, but their integration into current pathways of care will require a paradigm shift in the clinical management of patients with GBM.

\section{Financial \& competing interests disclosure}

The author has no relevant affiliations or financial involvement with any organization or entity with a financial interest in or financial conflict with the subject matter or materials discussed in the manuscript. This includes employment, consultancies, honoraria, stock ownership or options, expert testimony, grants or patents received or pending, or royalties.

No writing assistance was utilized in the production of this manuscript.

\section{References}

1 Greaves M, Maley CC. Clonal evolution in cancer. Nature 481(7381), 306-313 (2012).

2 Szerlip NJ, Pedraza A, Chakravarty D et al. Intratumoral heterogeneity of receptor tyrosine kinases EGFR and PDGFRA amplification in glioblastoma defines subpopulations with distinct growth factor response. Proc. Natl Acad. Sci. USA 109(8), 3041-3046 (2012).

3 Snuderl M, Fazlollahi L, Le LP et al. Mosaic amplification of multiple receptor tyrosine kinase genes in glioblastoma. Cancer Cell 20(6), 810-817 (2011).

4 Sottoriva A, Spiteri I, Piccirillo SG et al. Intratumor heterogeneity in human glioblastoma reflects cancer evolutionary dynamics. Proc. Natl Acad. Sci. USA 110(10), 4009-4014 (2013).

5 Shapiro JR, Yung WK, Shapiro WR. Isolation, karyotype, and clonal growth of heterogeneous subpopulations of human malignant gliomas. Cancer Res. 41(6), 2349-2359 (1981).

6 Sidransky D, Mikkelsen T, Schwechheimer K, Rosenblum ML, Cavanee W, Vogelstein B. Clonal expansion of p53 mutant cells is associated with brain tumour progression. Nature 355(6363), 846-847 (1992).

7 Meyerson M, Gabriel S, Getz G. Advances in understanding cancer genomes through second-generation sequencing. Nat. Rev. Genet. 11(10), 685-696 (2010).
8 Yates LR, Campbell PJ. Evolution of the cancer genome. Nat. Rev. Genet. 13(11), 795-806 (2012).

9 Brennan CW, Verhaak RG, McKenna A et al. The somatic genomic landscape of glioblastoma. Cell 155(2), 462-477 (2013).

10 Gerlinger M, Rowan AJ, Horswell S et al. Intratumor heterogeneity and branched evolution revealed by multiregion sequencing. N. Engl. J. Med. 366(10), 883-892 (2012).

11 Diaz LA, Jr., Williams RT, Wu J et al. The molecular evolution of acquired resistance to targeted EGFR blockade in colorectal cancers. Nature 486(7404), 537-540 (2012). 
12 Mcgranahan N, Swanton C. Biological and therapeutic impact of intratumor heterogeneity in cancer evolution. Cancer Cell 27(1), 15-26 (2015).

13 Weller M, Wick W, Von Deimling A. Isocitrate dehydrogenase mutations: a challenge to traditional views on the genesis and malignant progression of gliomas. Glia 59(8), 1200-1204 (2011).

14 Piccirillo SG, Spiteri I, Sottoriva A et al. Contributions to drug resistance in glioblastoma derived from malignant cells in the sub-ependymal zone. Cancer Res. 75(1), 194-202 (2015).
15 Patel AP, Tirosh I, Trombetta JJ et al. Single-cell RNA-seq highlights intratumoral heterogeneity in primary glioblastoma. Science 344(6190), 1396-1401 (2014).

16 Nathanson DA, Gini B, Mottahedeh J et al. Targeted therapy resistance mediated by dynamic regulation of extrachromosomal mutant EGFR DNA. Science 343(6166), 72-76 (2014).

17 Stupp R, Hegi ME, Gilbert MR, Chakravarti A. Chemoradiotherapy in malignant glioma: standard of care and future directions. J. Clin. Oncol. 25(26), 4127-4136 (2007).
18 Yip S, Miao J, Cahill DP et al. MSH6 mutations arise in glioblastomas during temozolomide therapy and mediate temozolomide resistance. Clin. Cancer Res. 15(14), 4622-4629 (2009).

19 Johnson BE, Mazor T, Hong C et al. Mutational analysis reveals the origin and therapy-driven evolution of recurrent glioma. Science 343(6167), 189-193 (2014).

$20 \mathrm{Kim} \mathrm{H}$, Zheng S, Amini SS et al. Wholegenome and multisector exome sequencing of primary and post-treatment glioblastoma reveals patterns of tumor evolution. Genome Res. 25(3), 316-327 (2015). 